\title{
Modelo Matemático Sazonal para Malária
}

\author{
A.P.P. WYSE ${ }^{1}$, L. BEVILACQUA ${ }^{2}$, Laboratório Nacional de Computação Cientí- \\ fica, LNCC, Av. Getúlio Vargas 333, 25651-075 Petrópolis, RJ, Brasil. \\ M. RAFIKOV 3 , Universidade Regional do Noroeste do RS, UNIJUÍ, Rua \\ São Francisco 501, 98700-000 Ijuí, RS, Brasil.
}

Resumo. Na Amazônia, onde se concentram 99\% dos casos de malária no Brasil, ocorre uma variação nos índices de incidência da malária no decorrer do ano, a qual está relacionada com as condições climáticas que afetam o desenvolvimento do vetor. Nesse trabalho, apresentamos um modelo matemático representado por um sistema de equações diferenciais ordinárias o qual descreve a dinâmica da relação humanovetor contemplando características sazonais e diferentes níveis de tratamento.

\section{Introdução}

A malária é uma doença infecciosa causada por um protozoário do gênero Plasmodium que é transmitido de pessoa infectada a pessoa sadia através da picada de fêmeas de mosquitos do gênero Anopheles.

O maior número de casos relatados no continente americano ocorre no Brasil, que registra cerca de 500 mil casos; destes, 99\% se concentram na região amazônica.[10] A malária afeta principalmente pessoas pobres e que vivem em áreas rurais onde o atendimento médico é inexistente ou precário. Esse fato motivou a diferenciação no tratamento que é contemplada neste artigo. Os danos econômicos atribuídos a malária classificam esta doença como uma das quatro principais causas de pobreza no mundo [10].

A incidência de malária na Amazônia, assim como em outras regiões tropicais, sofre variações com as estações do ano. Nessas regiões a temperatura é praticamente estável, mas os índices de umidade variam conforme a época do ano, assim o ritmo de propagação da malária se dá de acordo com as chuvas, e a estiagem diminui a proliferação de mosquitos contribuindo para o decréscimo do número de casos da doença.

O principal transmissor de malária no Brasil é o mosquito Anopheles darlingi. Ele tem como criadouro grandes volumes de água, sendo o principal vetor da região amazônica. Sua sazonalidade está relacionada ao nível das águas dos rios e aos períodos de chuvas e secas. O aumento das chuvas resulta em uma elevação da

\footnotetext{
${ }^{1}$ anawyse@lncc.br

${ }^{2}$ bevi@lncc.br

${ }^{3}$ rafikov@unijui.tche.br
} 
quantidade de mosquitos, ocasionando ondas epidêmicas. Por outro lado, as fortes chuvas podem arrastar os mosquitos para locais inadequados, destruindo os criadouros, o que resulta em um declínio da incidência de malária. Assim, o período de maior densidade de mosquitos coincide com a estabilização dos seus criadouros, logo após as fortes chuvas [8].

Modelos matemáticos têm sido desenvolvidos no intuito de melhor compreender a dinâmica envolvendo a população de mosquitos e a transmissão de malária. $\mathrm{Na}$ seção seguinte, propomos um sistema não autônomo de equações diferenciais ordinárias não lineares que modela matematicamente a dinâmica da transmissão da malária considerando os diferentes níveis de tratamento acessíveis às pessoas infectadas e os fatores sazonais que afetam o desenvolvimento do vetor. A seção 2.1 trata da estimação de parâmetros para o modelo matemático previamente descrito, usando informações disponíveis na literatura relacionada à Amazônia. Na seção 2.2 são apresentados alguns experimentos numéricos considerando três grupos de pessoas infectadas sujeitas à três níveis de intensidade de tratamento. Na seção 3 são discutidas algumas questões relevantes sobre a importância do investimento em saúde.

\section{Modelo Matemático}

O modelo matemático descrito a seguir considera a dinâmica do processo infeccioso da malária em regiões onde a variação sazonal na densidade populacional de mosquitos é um fator fundamental. As equações que compõe o modelo descrevem a variação das respectivas populações ao longo do tempo.

Para sua elaboração, as seguintes considerações devem ser feitas:

- O modelo proposto considera duas populações: $H$ - Humanos, com tamanho constante e $V$ - Mosquitos, com tamanho variável. A hipótese de que o tamanho da população de humanos é constante enquanto o tamanho da população de mosquitos é variável se deve ao fato de que uma geração de humanos corresponde a muitas gerações de mosquitos em função da diferença entre suas expectativas de vida;

- Na população de humanos são consideradas pessoas de todas as idades e ambos os sexos. Na população de mosquitos são consideradas apenas as fêmeas adultas, pois apenas estas alimentam-se de sangue a fim de obterem proteína necessária para a maturação de seus ovos;

- Os fenômenos migratórios são desprezíveis;

- Cada uma das populações é dividida em três compartimentos que representam as variáveis de estado: Suscetíveis $\left(H_{s}\right.$ e $\left.V_{s}\right)$, Expostos $\left(H_{e}\right.$ e $\left.V_{e}\right)$ e Infecciosos $\left(H_{i}\right.$ e $\left.V_{i}\right)$, onde $H=H_{s}+H_{e}+H_{i}$ e $V=V_{s}+V_{e}+V_{i}$;

- É considerada apenas a transmissão humano-mosquito-humano, não sendo encontrado na literatura nenhum relato a respeito da transmissão de malária humana por outros animais;

- A doença não confere nenhuma imunidade, o humano curado retorna imediatamente à classe dos suscetíveis. Essa hipótese é justificável nos casos em que ocorre um grande índice de reincidência da doença em humanos, como é observado na Amazônia; 
- A doença não reduz a fecundidade em humanos ou mosquitos;

- A mortalidade atribuída a doença é desconsiderada por ser muito baixa na região em questão;

- Todos os seres (humanos ou mosquitos) nascem suscetíveis;

- Os mosquitos suscetíveis, expostos e infecciosos picam com a mesma frequência;

- A taxa de emergência de fêmeas para a classe adulta é assumida totalmente dependente da densidade, enquanto que a taxa de mortalidade de mosquitos é assumida parcialmente dependente da densidade;

- O tempo de recuperação dos humanos depende da eficácia do tratamento. São consideradas $n$ situações: pessoas tratadas eficazmente, pessoas cujo tratamento foi incompleto ou inadequado ao tipo de Plasmodium que elas adquiriram e tantas outras situações quantas se queira especificar. Para os mosquitos, bem como para os humanos que nunca foram tratados, o período infeccioso cessa com o fim da sua vida;

- A taxa de recrutamento de fêmeas para a fase adulta e a capacidade suporte são parâmetros dependentes do tempo.

Desta forma, o modelo que descreve a dinâmica da malária pode ser posto nos seguintes termos:

$$
\left\{\begin{array}{l}
\frac{d H_{s}(t)}{d t}=\mu H(t)-a b V_{i}(t) \frac{H_{s}(t)}{H(t)}-\mu H_{s}(t)+\sum_{j=1}^{n}\left(\phi_{j} p_{j}\right) H_{i}(t) \\
\frac{d H_{e}(t)}{d t}=a b V_{i}(t) \frac{H_{s}(t)}{H(t)}-\mu H_{e}(t)-\eta H_{e}(t) \\
\frac{d H_{i}(t)}{d t}=\eta H_{e}(t)-\mu H_{i}(t)-\sum_{j=1}^{n}\left(\phi_{j} p_{j}\right) H_{i}(t) \\
\frac{d V_{s}(t)}{d t}=\varepsilon(t) V(t)-\frac{\left(\varepsilon(t)-\delta_{1}\right)}{k(t)} V_{s}(t) V(t)-a c V_{s}(t) \frac{H_{i}(t)}{H(t)}-\left(\delta_{1}+\delta_{2}\right) V_{s}(t) \\
\frac{d V_{e}(t)}{d t}=a c V_{s}(t) \frac{H_{i}(t)}{H(t)}-\frac{\left(\varepsilon(t)-\delta_{1}\right)}{k(t)} V_{e}(t) V(t)-\left(\delta_{1}+\delta_{2}\right) V_{e}(t)-\alpha V_{e}(t) \\
\frac{d V_{i}(t)}{d t}=\alpha V_{e}(t)-\frac{\left(\varepsilon(t)-\delta_{1}\right)}{k(t)} V_{i}(t) V(t)-\left(\delta_{1}+\delta_{2}\right) V_{i}(t),
\end{array}\right.
$$

onde $\mu$ é a taxa de natalidade e mortalidade para humanos por u.t., as quais são idênticas pela hipótese de que a população de humanos é constante e as taxas de migração não foram consideradas; $a$ é o número de picadas em humanos por mosquito por u.t.; $b$ é a probabilidade de que um humano suscetível picado por um mosquito infeccioso desenvolva a infecção; $c$ é a probabilidade de que um mosquito suscetível desenvolva a infeção ao picar um humano infeccioso; $\eta$ e $\alpha$ são as taxas de transição do compartimento dos expostos para o compartimento dos infecciosos para humanos e mosquitos por u.t., respectivamente; $p_{j}$ é a proporção da população infectada submetida ao tratamento $j$, onde $\sum_{j=1}^{n} p_{j}=1 ; \phi_{j}$ são as taxas de recuperação para $p_{j}$ por u.t.; $\varepsilon(t)$ é uma função continuamente diferenciável variando no intervalo $\left[\varepsilon_{\min }, \varepsilon_{\max }\right]$ que representa a taxa de recrutamento de fêmeas para a classe adulta no tempo $t ; k(t)$ é uma função continuamente diferenciável variando 
no intervalo $\left[k_{\min }, k_{\max }\right]$ que representa a capacidade suporte no tempo $t ; \delta_{1}$ é a taxa de mortalidade dos mosquitos dependente da densidade por u.t. e $\delta_{2}$ é a taxa de mortalidade dos mosquitos independente da densidade por u.t..

As equações que descrevem o comportamento das populações totais de humanos são

$$
\left\{\begin{array}{l}
\frac{d H(t)}{d t}=0 \\
\frac{d V}{d t}=\left(\varepsilon(t)-\delta_{1}-\delta_{2}\right) V(t)-\frac{\left(\varepsilon(t)-\delta_{1}\right)}{k(t)} V(t)^{2},
\end{array}\right.
$$

onde a população total de humanos é constante conforme a hipótese do modelo e a população total de mosquitos é uma generalização do modelo de crescimento logístico de Verhulst para o caso sazonal.

\subsection{Estimativas}

O modelo matemático introduzido anteriormente visa representar características da Amazônia, descrevendo a flutuação sazonal que ocorre na densidade de mosquitos nesta região. As seguintes funções são uma boa aproximação:

$$
\varepsilon(t)=\frac{1}{\varepsilon^{-1}+\varepsilon_{0} \cos \left(\frac{\pi}{6} t\right)} ; k(t)=\frac{1}{k^{-1}+k_{0} \cos \left(\frac{\pi}{6} t\right)} .
$$

Note que a densidade máxima de mosquitos ocorre no mês de Junho. [8] Os parâmetros foram estimados considerando mês como unidade de tempo.

A funções de sazonalidade escolhidas apresentam o seguinte comportamento:

- Para $t=0+12 n(n \in \aleph)$, a taxa de recrutamento e a capacidade suporte atingem seus valores mínimos: $\frac{1}{\varepsilon^{-1}+\varepsilon_{0}}$ e $\frac{1}{k^{-1}+k_{0}}$, respectivamente;

- Para $t=3+6 n(n \in \aleph)$, a taxa de recrutamento e a capacidade suporte atingem seus valores médios: $\varepsilon$ e $k$, respectivamente;

- Para $t=6+12 n(n \in \aleph)$, a taxa de recrutamento e a capacidade suporte atingem seus valores máximos: $\frac{1}{\varepsilon^{-1}-\varepsilon_{0}} \mathrm{e} \frac{1}{k^{-1}-k_{0}}$, respectivamente.

O valor de $\varepsilon$ é estimado considerando o fato de que a população de mosquitos cresce exponencialmente na ausência de qualquer fator limitante, sendo seu crescimento descrito nesse caso por $\frac{d V(t)}{d t}=\varepsilon V(t)$, cuja solução é dada por $V(t)=V(0) e^{\varepsilon t}$, de onde se conclui que $\varepsilon=\frac{1}{t} \ln \left(\frac{V(t)}{V(0)}\right)$.

Estudos de laboratório de Santos et.al. [7] a respeito da biologia de Anopheles darlingi mostraram que o ciclo biológico de ovo a adulto leva, em média, 15,6 dias (0,51 meses) para se completar com uma taxa de sobrevivência de $57 \%$. O número médio de ovos a cada postura é de 110 ovos [7] e cada fêmea põe ovos em média duas vezes em sua vida [8]. Considerando que $50 \%$ dos ovos resultam em fêmeas, então: 


$$
\varepsilon=\frac{1}{0,51} \ln \left(\frac{2 \times 110 \times 0,5 \times 0,57}{1}\right)=8,114 / \text { mês. }
$$

O valor de $\left(\delta_{1}+\delta_{2}\right)$ é estimado considerando o fato de que a população de mosquitos decresce exponencialmente na ausência de qualquer fator de crescimento, sendo sua variação descrito por $\frac{d V(t)}{d t}=-\left(\delta_{1}+\delta_{2}\right) V(t)$, cuja solução é dada por $V(t)=V(0) e^{-\left(\delta_{1}+\delta_{2}\right) t}$, de onde se conclui que $\delta_{1}+\delta_{2}=\frac{1}{t} \ln \left(\frac{V(t)}{V(0)}\right)$.

Charlwood e Alecrim [2] estimaram em 80,4\% a taxa diária de sobrevivência dos mosquitos adultos. Então,

$$
\delta_{1}+\delta_{2}=\frac{1}{1} \ln \left(\frac{0,804^{30}}{1}\right)=6,545 / \text { mês. }
$$

Dados $\varepsilon$ e $\delta_{1}+\delta_{2}$, o problema é encontrar $\varepsilon_{0}$ tal que a taxa intrínseca de crescimento das fêmeas adultas de mosquito seja sempre positiva. Podemos dizer que se essa condição for satisfeita para $t=0+12 n(n \in \aleph)$, ela será satisfeita para todo $t \in \Re^{+}$. Então,

$$
\frac{1}{\varepsilon^{-1}+\varepsilon_{0}}>\delta_{1}+\delta_{2} \Rightarrow \varepsilon_{0}<\frac{1}{\delta_{1}+\delta_{2}}-\frac{1}{\varepsilon}
$$

Conseqüentemente, $\varepsilon_{0}<0,00295$.

Assumindo que a expectativa média de vida da população humana seja 60 anos, temos que $\mu=\frac{1}{720}=0,0014 /$ mês.

A taxa de picada do Anopheles darlingi pode ser estimada como a razão entre a proporção de sangue humano ingerida pelo mosquito, designada por hbi, e a duração do ciclo gonotrófico, designado por gc, que corresponde ao intervalo entre as refeições do mosquito. [6] [5] Então, $a=\frac{h b i}{g c}$.

O mosquito Anopheles darlingi se alimenta de sangue humano em uma proporção de 45,8\% [4] e suas refeições são feitas em média a cada 2,3 dias [2]. Logo,

$$
a=\frac{0,458}{2,3} \times 30 \Rightarrow a=5,974 / \text { mês. }
$$

Os coeficientes $b$ e $c$ devem variar no intervalo $[0,1]$ conforme o grau de suscetibilidade de humanos e mosquitos, respectivamente. É importante salientar que o plasmodium só se desenvolve no corpo do mosquito se gametócitos de ambos sexos forem ingeridos durante a picada. [1] [6]. Nas simulações numéricas a seguir assumiremos $b=c=0,3$, logo que esses parâmetros não foram encontrados na literatura.

O período latente para malária é definido como o intervalo de tempo desde a picada até o surgimento de gametócitos no sangue (período latente intrínseco - para humanos) ou o surgimento de esporozoítos nas glândulas salivares (período latente extrínseco - para mosquitos). Estima-se, em média, um período latente intrínseco de 10 dias (0,33 mês) para Plasmodium falciparum e Plasmodium vivax.[1] [6] A duração do período latente extrínseco varia com a temperatura ambiente, sendo 
aproximadamente 6 dias para uma temperatura média de $27^{\circ} \mathrm{C}$.[1] Assim, a taxa de transição do compartimento dos expostos para o compartimento dos infecciosos para humanos e mosquitos é, respectivamente

$$
\eta=\frac{1}{0,33}=3 / \text { mês; } \alpha=\frac{1}{0,1667}=6 / \text { mês. }
$$

As pessoas infectadas recebem tratamento diferenciado dependendo das condições socioeconomicas da região e do nível de informação a respeito das conseqüências da doença. Em relação ao tratamento, são consideradas aqui três situações:

- Tratamento completo e eficaz: Neste caso o indivíduo permanece infeccioso por um período que vai desde o término do período latente até a total eliminação da doença em seu organismo. Esse espaço de tempo compreende o intervalo entre o início do tratamento e a cura, que é de aproximadamente 15 dias somado ao período que corresponde a diferença entre o período de incubação (período assintomático) e o período latente (período de não transmissibilidade), pois o indivíduo só procura tratamento ao manifestar os sintomas da doença. Logo, $\phi_{1}=\frac{1}{15+5} \times 30=1,5 /$ mês;

- Tratamento incompleto: Neste caso o indivíduo permanece infeccioso por um longo período, pois o seu organismo vai eliminando a doença de forma lenta e gradual, assim como é administrado o seu tratamento. Nesse caso o período infeccioso dure de 1 a 3 anos.[9] Para a realização das simulações numéricas vamos considerar o período infeccioso de um ano. Então $\phi_{2}=\frac{1}{12}=0,0832 / \mathrm{mês}$;

- Tratamento inexistente: Quando o tratamento não é realizado, o indivíduo permanece infeccioso até o fim de sua vida. Isso ocorre freqüentemente nos casos em que o paciente infectado é assintomático. Neste caso, $\phi_{3}=0$.

Os parâmetros $k$ e $k_{0}$ são obtidos de forma a melhor ajustar os dados de campo. Neste caso são assumidos $k=85$ e $k_{0}=0,00975$, que nós ajustamos para o município de Novo Airão/AM, Brasil.[8]

O número de mosquitos fêmea por pessoa é definido como a razão entre o número de picadas por humano por u.t. e a taxa de picada.[6] [5] Para Novo Airão/AM foi obtido através de pesquisas de campo um índice de picada por homem/hora (IPHH) de 0,096.[8] Então,

$$
m=\frac{I P H M}{a}=\frac{0,096 \times 24 \times 30}{5,974}=11,57 \text { mosquitos fêmea } / \text { humano. }
$$

Com base nos dados coletados em Novo Airão/AM [8], o número total de mosquitos fêmea coletados durante o ano de 1997 foi 804. Temos então que

$$
\frac{\sum_{\text {mes }=\text { Janeiro }}^{\text {Dezembro }} V(\text { mes })}{12 \times H(t)}=11,57 \text { ou } H(t)=\frac{804}{12 \times 11,57}=5,8 \text { humanos. }
$$


Como a população humana é assumida constante, podemos assumir a condição inicial $H(0)=5,8$. A evolução da população não é sensível à distribuição entre as três categorias (suscetíveis, expostos e infecciosos). De acordo com os dados de campo [8], a condição inicial para a população de mosquitos é definida $V(0)=6$, já que estamos considerando o instante inicial como o começo do ano.

\subsection{Simulações Numéricas}

As simulações numéricas visam verificar a dinâmica sazonal descrita pelo modelo, cujo sistema foi resolvido usando o método de diferenças finitas Runge-Kutta de quarta ordem.[3] Os coeficientes utilizados nas simulações foram estimados previamente. As taxas de mortalidade do mosquito foram consideradas $\delta_{1}=4 \mathrm{e}$ $\delta_{2}=2,545$.

A população total de mosquitos apresenta conformidade com as observações de campo realizadas em 1997 no município de Novo Airão/AM [8] e sua evolução é vista na Figura 1, onde é possível perceber a influência da variação sazonal na sua densidade populacional.

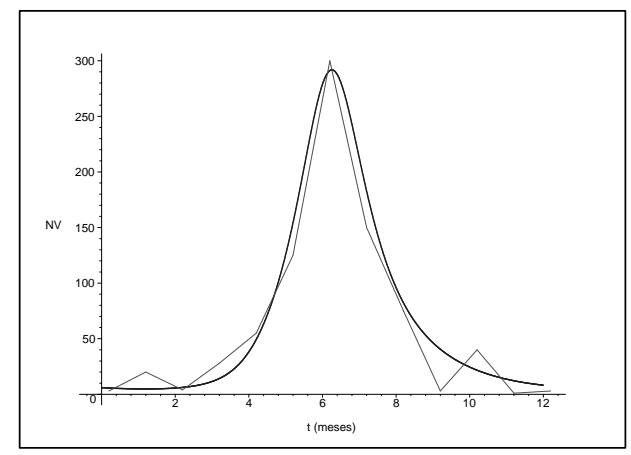

Figura 1: Evolução temporal do An. darlingi: Simulação numérica (curva preta) e dados de campo (curva cinza)

Considerando inicialmente o caso mais extremo, que seria nenhum tipo de tratamento aos infectados com malária, vimos na Figura 2 que a quantidade de infectados se eleva gradativamente até o mês de Junho, onde atinge o ponto máximo. Nesse momento todos os humanos já contraíram a doença e, por falta de tratamento, permanecerão hospedeiros do protozoário até o fim da sua vida. Em relação aos mosquitos, no ponto máximo estão infectados apenas 5\%; isto porque o ciclo de vida do mosquito é curto e ele morre pouco tempo após tornar-se infeccioso. Como todos nascem suscetíveis, poucos mosquitos infectados são encontrados.

Os cenários mostrados na Figura 3 consideram uma proporção da população de infecciosos recebendo tratamento eficaz e adequado. Nesse caso $70 \%$ da população infectada recebeu o melhor tratamento, $10 \%$ receberam tratamento incompleto e $20 \%$ não receberam tratamento. Embora tenha havido uma drástica redução da incidência de malária, o tratamento ainda não foi suficiente para eliminar a doença na região, quando a população atinge seu equilíbrio (cíclico) são observados índices 


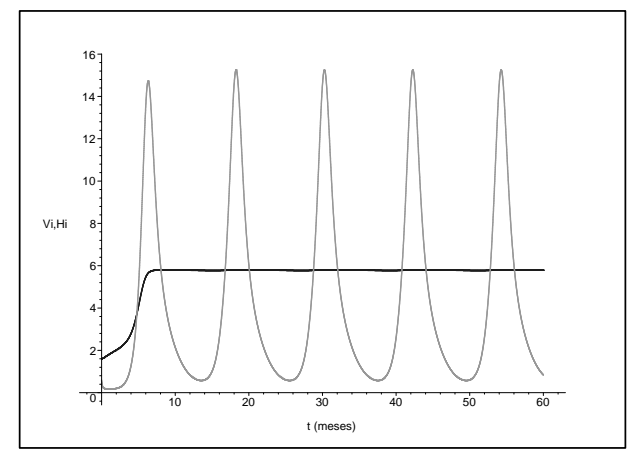

Figura 2: População de humanos infectados (curva preta) e mosquitos infectados (curva cinza). Foram admitidos $p_{1}=0, p_{2}=0$ e $p_{3}=1$.

máximos de 1,45 humanos infecciosos e 1,6 mosquitos infecciosos. Apesar da malária ainda estar presente na população, sua incidência é baixa, correspondendo a $25 \%$ de pessoas infectadas $0,53 \%$ de mosquitos infectados no período mais crítico.

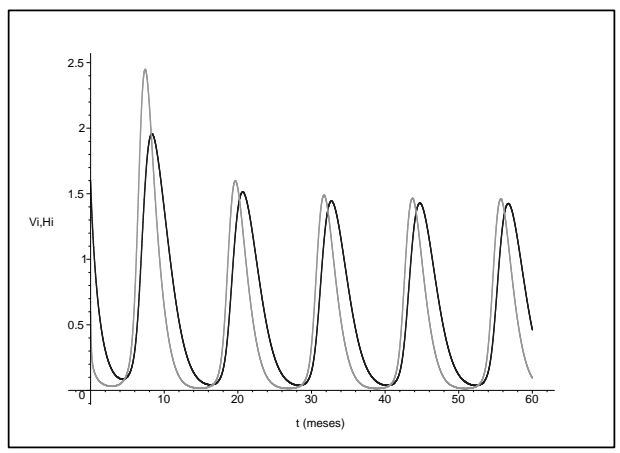

Figura 3: População de humanos infectados (curva preta) e mosquitos infectados (curva cinza). Foram admitidos $p_{1}=0,7, p_{2}=0,1$ e $p_{3}=0,2$.

Aumentando a proporção da população que recebe tratamento completo e eficaz para $88 \%$ obtemos um resultado bastante satisfatório, como pode ser visto na Figura 4. A proporção de $10 \%$ para tratamento incompleto vem sendo mantida e os $2 \%$ restantes de pessoas infectadas não recebem tratamento. Para os parâmetros utilizados aqui e um programa de tratamento conforme o que foi indicado na simulação numérica da Figura 4 é possível eliminar completamente a malária na região após 5 anos de investimento em tratamento.

\section{Discussão}

O modelo contemplando variação sazonal da densidade de mosquitos considerado neste trabalho descreve de forma mais realista a dinâmica populacional de mosquitos em áreas como a Amazônia. A conformidade do modelo com as observações 


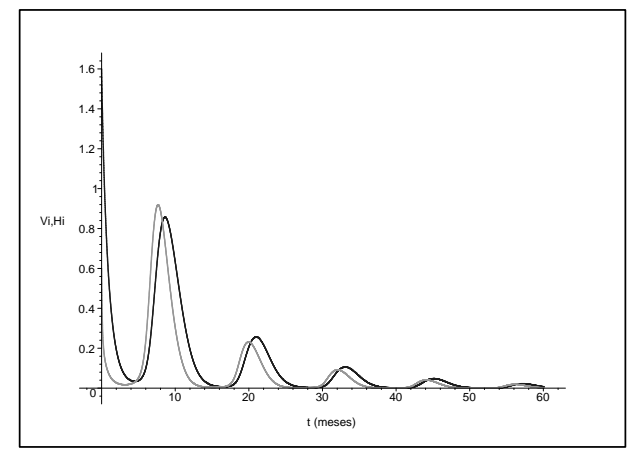

Figura 4: População de humanos infectados (curva preta) e mosquitos infectados (curva cinza). Foram admitidos $p_{1}=0,8, p_{2}=0,1$ e $p_{3}=0,1$.

da região indicam que ele é adequado tanto para esta região como para outras que tenham as mesmas características. Considerando o fato de que um modelo matemático é apenas uma aproximação da realidade, mostramos que o modelo aqui proposto descreve muito bem a evolução da população de mosquitos ao longo do tempo e sua relação com as ondas epidêmicas anuais observadas em todas as regiões da Amazônia, sendo adequado para fornecer aos programas de combate à malária diretrizes que indiquem a forma de tratamento mais adequada para reduzir a incidênciada desta doença.

Conforme se verificou nas simulações numéricas, o investimento em saúde tem um papel fundamental na redução da incidência de malária, indicando uma tendência à erradicação ao longo do tempo. Um estudo dos custos deste tratamento não foi feito neste trabalho e nem era nosso objetivo, mas os cenários obtidos através das simulações numéricas mostram que as despesas com tratamento são reduzidas a cada ano devido ao fato de que cada vez mais o número de infectados decresce. Há de se pensar no custo-benefício que o investimento em saúde trará para o município, a morbidade que a doença provoca induz a um decréscimo significativo da produção material e intelectual pelo fato de que o indivíduo infectado se ausenta do trabalho e da escola. A exploração do potencial turístico da região também é afetada.

As pesquisas de campo/laboratório e a interação entre pesquisadores de diferentes áreas do conhecimento devem ser intensificadas no sentido de obter informações mais precisas e atualizadas. Dessa forma, os cenários traçados poderão ser mais próximos à realidade para que tenham condições de fornecer diretrizes aos órgãos relacionados à saúde pública, indicando meios mais eficazes e econômicos de controlar a doença.

\section{Agradecimentos}

Ao CNPq e à Rede GEOMA pelo financiamento desta pesquisa. Ao Dr. Manuel Cesário (UFAC) pela discussão sobre o tratamento da malária e aos revisores anônimos por suas contribuições para este artigo. 
Abstract. In the Amazônia, where are concentrated $99 \%$ of malaria cases in Brazil, it occurs a variation of the malaria incidence on the year, which is related to climatic conditions that affect the vector development. In this work, we present a mathematical model represented by a system of ordinary differential equations which describes the dynamic human-vector regarding seasonal peculiarities and different treatment levels.

\section{Referências}

[1] R.M. Anderson, R.M. May, "Infectious Diseases of Humans", Oxford Science Publication, New York, 1991.

[2] J.D. Charlwood, W.A. Alecrim, Capture-recapture studies with the South America malaria vector Anopheles darlingi, Ann. Trop. Med. Parasitol., 83 (1989), 569-576.

[3] W.E. Grove, "Brief Numerical Methods", Prentice-Hall, 1966.

[4] A. Kiszewski et al., A Global Index Representing the Stability of Malaria Transmission, Am. J. Trop. Med. Hyg., 70, No. 5 (2004), 486-498.

[5] H.-I Lee et al., Malaria transmission potential by Anopheles sinensis in the Republic of Korea, The Korean Journal of Parasitology, 39, No. 2 (2001), 185-192.

[6] L. Molineaux, G. Gramiccia, "The Garki Project: Research on the epidemiology and control of malaria in the sudan savanna of West Africa", WHO, Geneva, 1980.

[7] J. Santos et al., Biologia de anofelinos amazônicos 1- Ciclo biológico, postura e estádios larvais de Anopheles darlingi Root 1926 (Díptera: Culicidae) da Rodovia Manaus - Boa Vista, Acta Amazônica, 11, No. 4 (1981), 789-797.

[8] W. Tadei et al., Entomologia da Malária em Áreas de Colonização da Amazônia, Programa de Pesquisa Dirigida - PPD, MCT, 1997.

[9] Programa Nacional de Prevenção e Controle da Malária, Brasília: Ministério da Saúde. Fundação Nacional da Saúde - FUNASA, 2002.

[10] Roll Back Malaria Department, Basic Facts on Malaria, World Health Organization, 2004. 